\title{
Performance Evaluation of G8, a High-Sensitivity Benchtop Preclinical PET/CT Tomograph
}

\author{
Zheng Gu ${ }^{1,2}$, Richard Taschereau ${ }^{1}$, Nam T. Vu ${ }^{2}$, David L. Prout ${ }^{1}$, Robert W. Silverman ${ }^{1}$, Jason T. Lee ${ }^{1,3}$, and \\ Arion F. Chatziioannou ${ }^{1-3}$ \\ ${ }^{1}$ Crump Institute for Molecular Imaging, David Geffen School of Medicine, UCLA, Los Angeles, California; ${ }^{2}$ Sofie Biosciences, \\ Culver City, California; and ${ }^{3}$ Jonsson Comprehensive Cancer Center, UCLA, Los Angeles, California
}

\begin{abstract}
G8 is a benchtop integrated PET/CT scanner dedicated to highsensitivity and high-resolution imaging of mice. This work characterizes its National Electrical Manufacturers Association NU 4-2008 performance where applicable and also assesses the basic imaging performance of the CT subsystem. Methods: The PET subsystem in G8 consists of 4 flat-panel detectors arranged in a boxlike geometry. Each panel consists of 2 modules of a $26 \times 26$ pixelated bismuth germanate scintillator array with individual crystals measuring $1.75 \times$ $1.75 \times 7.2 \mathrm{~mm}$. The crystal arrays are coupled to multichannel photomultiplier tubes via a tapered, pixelated glass lightguide. A conebeam CT scanner consisting of a MicroFocus x-ray source and a complementary metal oxide semiconductor detector provides anatomic information. Sensitivity, spatial resolution, energy resolution, scatter fraction, count-rate performance, and the capability of performing phantom and mouse imaging were evaluated for the PET subsystem. Noise, dose level, contrast, and resolution were evaluated for the CT subsystem. Results: With an energy window of 350-650 keV, the peak sensitivity was $9.0 \%$ near the center of the field of view. The crystal energy resolution ranged from $15.0 \%$ to $69.6 \%$ in full width at half maximum (FWHM), with a mean of $19.3 \%$ $\pm 3.7 \%$. The average intrinsic spatial resolution was 1.30 and $1.38 \mathrm{~mm}$ FWHM in the transverse and axial directions, respectively. The maximum-likelihood expectation maximization reconstructed image of a point source in air averaged $0.81 \pm 0.11 \mathrm{~mm}$ FWHM. The peak noise-equivalent count rate for the mouse-sized phantom was $44 \mathrm{kcps}$ for a total activity of $2.9 \mathrm{MBq}(78 \mu \mathrm{Ci})$, and the scatter fraction was $11 \%$. For the CT subsystem, the value of the modulation transfer function at $10 \%$ was 2.05 cycles $/ \mathrm{mm}$. Conclusion: The overall performance demonstrates that the G8 can produce highquality images for molecular imaging-based biomedical research.
\end{abstract}

Key Words: small-animal imaging; PET/CT, performance evaluation; instrumentation; molecular imaging

J Nucl Med 2019; 60:142-149

DOI: 10.2967/jnumed.118.208827

$\mathbf{P}$

ET is a widely used imaging modality for noninvasive, in vivo studies of biologic processes and has found wide application in pharmacology, genetics, pathology, and oncology $(1,2)$. The

Received Feb. 2, 2018; revision accepted Jun. 12, 2018.

For correspondence or reprints contact: Zheng Gu, 570 Westwood Plaza,

Crump Institute for Molecular Imaging, Los Angeles, CA 90095.

E-mail: zhgu@mednet.ucla.edu

Guest Editor: David Townsend, National University of Singapore

Published online Jun. 14, 2018.

COPYRIGHT (c) 2019 by the Society of Nuclear Medicine and Molecular Imaging. demand for in vivo experiments with animal models of disease in translational research has been a driving force behind the advances in dedicated small-animal PET instruments $(3,4)$.

In molecular imaging applications, an anatomic reference is commonly required to help localize the molecular signal and assist quantification of tracer concentration $(5,6)$. CT is a modality that can provide fully tomographic anatomic information for better molecular signal localization (7). Volumetric CT data also facilitate tracer quantification by guiding corrections for attenuation (8), scattering of $\gamma$-energy in the object (9), and partial-volume effect (10). Besides, CT leads to accurate organ registration and image analysis when used with the mouse atlas registration algorithm developed at our institute (11).

G8 is an integrated PET/CT system implemented by Sofie Biosciences. It incorporates a cone-beam CT scanner and digital data acquisition electronics (PicoDigitizer). Compared with the previous G4 system $(12,13)$, each panel detector uses 2 extra rows of crystals at the transverse edges of the field of view (FOV), reducing the gap between detectors. Instead of 1-mm clear glass in $\mathrm{G} 4$, a tapered, pixelated lightguide is used in G8 to couple each crystal array to a single photomultiplier tube (PMT), improving scintillation light collection and its uniformity.

This work aims to characterize the overall performance of the G8 $\mathrm{PET} / \mathrm{CT}$ system. Studies including sensitivity, spatial resolution, energy resolution, scatter fraction, count-rate performance, and image quality were performed for the PET subsystem following the National Electrical Manufacturers Association (NEMA) NU 4-2008 standards (14) when possible. The noise, dose level, contrast, and spatial resolution were evaluated for the CT subsystem. In vivo rodent studies using radiolabeled tracers were acquired to demonstrate the capability of the G8 for high-sensitivity, high-resolution molecular imaging. Representative animal images are shown to emphasize the complementary nature of the molecular and anatomic information provided by this integrated PET/CT imaging platform.

\section{MATERIALS AND METHODS}

\section{System Description}

The G8 uses 4 opposing panel detectors placed at a face-to-face spacing of $5 \mathrm{~cm}$, arranged in a compact, boxlike geometry. The effective area of each panel is $47.44 \times 94.95 \mathrm{~mm}$, large enough to cover the whole body of a laboratory mouse (18-40 g). Each panel consists of 2 modules, and each module comprises a $26 \times 26$ pixelated bismuth germanate (BGO) scintillator array with individual crystals measuring $1.75 \times 1.75 \times 7.2 \mathrm{~mm}( \pm 0.05 \mathrm{~mm})$ and a pitch of $1.83 \mathrm{~mm}$ (Proteus). The 4 long sides of each individual crystal and the entrance surface of the scintillator array were bonded with specular optical reflector (3M). A tapered, pixelated glass lightguide was used 
to couple the crystal array to the photosensitive area of an H8500 multianode PMT (MAPMT; Hamamatsu Photonics). The scintillator array, lightguide, and MAPMT were permanently bonded with epoxy.

The front-end read-out electronics of each detector are integrated in a printed circuit board directly connected to the PMT. A chargedivision readout circuit is used to convert the 64 anode outputs from each MAPMT into energy and 2 position signals ( $x$ and $y$ ), whereas the timing signal is obtained directly from the 12th dynode on the PMT. The signals from all 8 detector modules (4 signals per module) are digitized by 32 free-running $125-\mathrm{MHz}$ analog-to-digital converters, at 14 bits per sample (PicoDigitizer). These digital samples are then processed in a Xilinx Virtex-6 field programmable gate array (Xilinx) in real time for event triggering, validation (15), and coincidence sorting. Energy discrimination is performed offline in software, during event histogramming. A delayed timing window is implemented in the field-programmable gate array to estimate random coincidence event rates (16).

All measurements in this work were postprocessed with an energy window of $350-650 \mathrm{keV}$ (17). The only exception was the sensitivity measurement, for which the lower energy threshold was varied between 150 and $350 \mathrm{keV}$ to show the influence of energy window on sensitivity. The coincidence timing window was set to $20 \mathrm{~ns}$. The acquired list-mode data were histogrammed into projection files, with delayed events subtracted to correct for random coincidences. Component-based normalization (18) was applied to compensate for the differences in individual detector efficiencies, estimated from measurements of a cylindric source filled with ${ }^{18} \mathrm{~F}$. The acceptance angles were not restricted by the system hardware, and all geometrically possible lines of response were acquired. For image reconstruction, the line of response with the largest acceptance angle crossing the FOV was $76^{\circ}$ in the transverse direction and $59^{\circ}$ in the axial direction. Fully 3-dimensional tomographic images were reconstructed by a maximum-likelihood expectation-maximization (MLEM) algorithm with incorporation of a system model based on a parameterized detector response (19). The system response matrix used in the MLEM reconstruction corresponds to symmetric cubic voxels with a side equal to one fourth the pitch of the crystals in the detector, and the cubic voxel size is $0.46 \times 0.46 \times 0.46 \mathrm{~mm}$. A total of 60 full iterations (no subsets) was used for image reconstruction, with no regularization and no postreconstruction smoothing. The rectangular system geometry precludes the use of conventional linear image reconstruction algorithms.

A cone-beam CT scanner consisting of a MicroFocus x-ray source (50 kV Magnum; Moxtek) and a complementary metal oxide semiconductor detector (Dexela 1512) was implemented to provide anatomic information, assisting PET signal localization and quantification. The CT scanner runs in continuous rotation mode and acquires 720 projections over one full $360^{\circ}$ rotation in about $1 \mathrm{~min}$. The $\mathrm{x}$-ray source operates at $50 \mathrm{kVp}$ with a maximum anode current of $200 \mu \mathrm{A}$. Images were reconstructed with a Feldkamp filtered backprojection algorithm (ramp filter) and a voxel size of $0.2 \mathrm{~mm}$ (20). The reconstructed FOV of the CT scanner was $5 \times 5 \times 10 \mathrm{~cm}$. The CT and PET subsystems in the G8 are mounted on a common gantry with a shared life-supporting chamber (21). The inherent coregistration of the functional PET images and anatomic CT images is achieved with a calibration step in a common spatial frame (22). A schematic of the G8 PET/CT scanner is shown in Figure 1, and the characteristics of the G4 and the G8 systems are summarized in Table 1.

\section{Energy Resolution}

A $0.22-\mathrm{MBq}{ }^{68} \mathrm{Ge}$ cylindric source (Eckert and Ziegler Isotope Products) with a diameter of $37 \mathrm{~mm}$ and a length of $100 \mathrm{~mm}$ was placed at the center of the field of view (CFOV) of the PET subsystem to acquire a 2-dimensional flood irradiation for each detector module. A semiautomated program defined the crystal look-up tables that

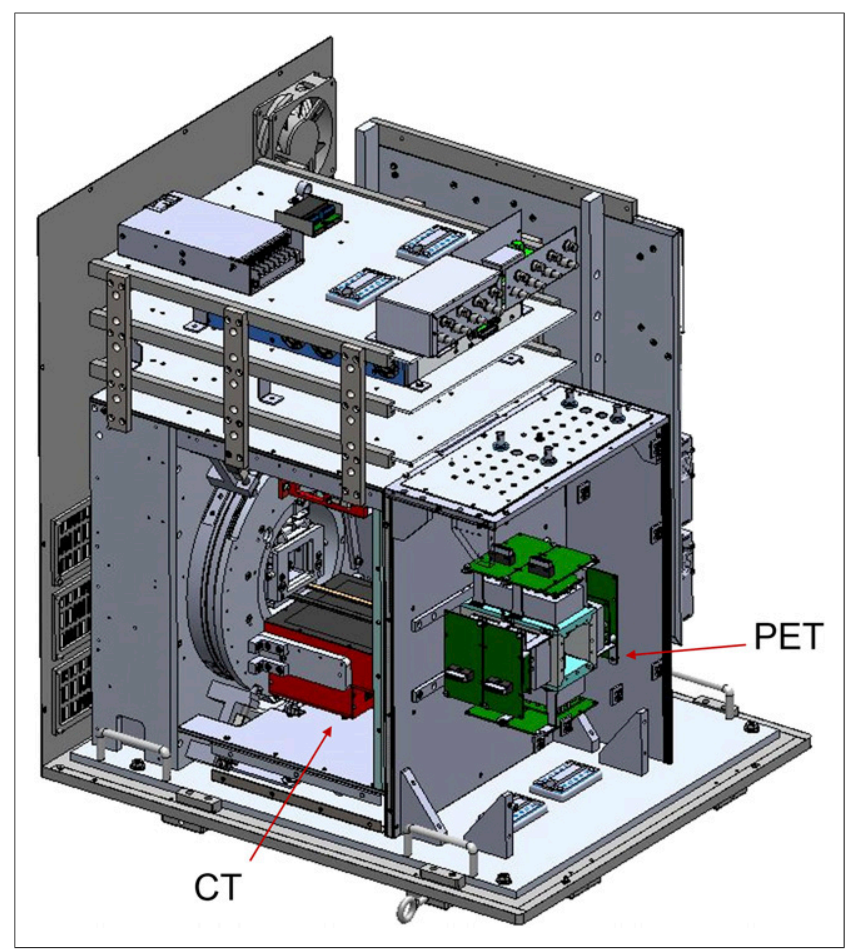

FIGURE 1. Schematic of G8 PET/CT tomograph.

classify regions in the flood image into the proper crystal of the scintillator array. Energy spectra of individual crystals were extracted on the basis of the look-up tables, and a gaussian function was fitted to the photopeak of each energy spectrum. Energy resolution was measured for every crystal in the scanner as the full width at half maximum (FWHM) of the gaussian function divided by the energy corresponding to the center of the photopeak, expressed as a percentage.

\section{Spatial Resolution}

A $0.34-\mathrm{MBq}{ }^{22} \mathrm{Na}$ point source with a nominal size of $0.3 \mathrm{~mm}$, embedded in a $1-\mathrm{cm}^{3}$ piece of acrylic was used (NEMA NU 4compliant, Eckert and Ziegler Isotope Products). Measurements were not corrected for the physical source dimensions, positron range, or noncollinearity of positron annihilation $\gamma$-energy.

Intrinsic Spatial Resolution. Starting from the CFOV of the PET subsystem, the point source was attached to a translation stage and stepped at $0.4-\mathrm{mm}$ steps across 13 crystals along the long axis of the FOV (half the axial length of a detector module). Because of geometric restriction by the side detectors, the point source could not be stepped across half the detector module along the short axis. Instead, the source was stepped across the central 14 crystals in a transverse plane of a detector module. Acquisition time was $60 \mathrm{~s}$ at each location. Coincidence counts for directly opposing crystal pairs were plotted as a function of source location. The count distribution of each crystal pair was fitted with a gaussian function, and the FWHM determined the intrinsic spatial resolution of the detectors.

Image Spatial Resolution. The point source was imaged at 2 axial locations: the axial CFOV and one fourth the axial FOV, $23.8 \mathrm{~mm}$ from the center along the axial direction. For each of these locations, the source was placed at $0,5,10$, and $15 \mathrm{~mm}$ from the geometric center along the transverse FOV. Acquisition time was $1 \mathrm{~min}$ at each position, and more than $10^{5}$ prompt counts were acquired per measurement. The NEMA NU 4 document also recommends measurements at 20 and $25 \mathrm{~mm}$ from the center, which were not included in this study because of the geometric restriction. Because of the box-shaped geometry of the 
TABLE 1

Characteristics of G4 and G8 Systems

\begin{tabular}{lcc}
\hline \multicolumn{1}{c}{ Characteristic } & G4 & G8 \\
\hline Crystal material & BGO & BGO \\
Crystal size & $1.75 \times 1.75 \times 7.2 \mathrm{~mm}$ & $1.75 \times 1.75 \times 7.2 \mathrm{~mm}$ \\
Crystal pitch & $1.83 \mathrm{~mm}$ & $1.83 \mathrm{~mm}$ \\
Crystal array & $24 \times 26$ crystals/PMT & $26 \times 26 \mathrm{crystals/PMT}$ \\
Light guide & 1-mm-thick glass layer & Tapered, pixelated light guide \\
PMT & Hamamatsu H8500 MAPMT & Hamamatsu H8500 MAPMT \\
Number of detector modules & 4 & 8 \\
Number of crystals per module & 1,248 & 676 \\
Number of crystals in total & 4,992 & 5,408 \\
Number of analog-to-digital converters & 16 & 32 \\
Signal-processing system & VHS-ADC (Lyrtech) \\
Anatomic reference approach & X-ray projection and optical photographic images & PicoDigitizer (Nutaq) \\
\hline
\end{tabular}

tomograph, conventional filtered backprojection reconstruction was not available and therefore, in a significant deviation from the NEMA protocol, images were reconstructed using the MLEM algorithm. As specified in the NEMA NU 4-2008 protocol, the response function was formed by summing 1-dimensional profiles that were parallel to the radial, tangential, and axial directions. A parabolic fit of the peak point and its 2 nearest neighboring points was used to determine the maximum value of the response function. Linear interpolation between adjacent pixels was used to determine the position of half and one tenth the parabolic curve maximum.

\section{Sensitivity}

A ${ }^{68} \mathrm{Ge}$ pointlike source (Eckert and Ziegler Isotope Products) embedded in thin steel tubing was used to measure absolute sensitivity. The thin steel casing ensures annihilation of all the positrons, with only nominal attenuation of the $511-\mathrm{keV} \gamma$-rays-an amount that was assumed to be insignificant in this study. The activity of the point source was $1.62 \mathrm{kBq}$ measured in a calibrated well-type $\gamma$-counter (Wallac Wizard 1480; Perkin Elmer). The activity was low enough to keep the counting losses to less than $1 \%$ and the randoms rate to less than $5 \%$ of the true event rate, fulfilling the NEMA NU 4-2008 recommendations. The axial sensitivity profile was measured with the ${ }^{68} \mathrm{Ge}$ source stepped from end to end of the axial FOV, with the axial positions of the source determined from the reconstructed images. The number of coincidences was recorded at each position for $60 \mathrm{~s}$. Delayed coincidences were subtracted from prompts before the true coincidences were divided by the actual source activity. This ratio was corrected for the branching ratio of ${ }^{68} \mathrm{Ga}(0.89)$, but the attenuation of the steel material surrounding the source was not compensated for. The average sensitivity for a mouse-sized object (with a 7-cm axial length) was calculated from the measured axial sensitivity profile.

\section{Scatter and Count-Rate Performance}

Count-rate performance was evaluated using the NEMA NU 4 mouse-sized phantom, which is a 70 -mm-long and 25 -mm-diameter solid cylinder made of high-density polyethylene $\left(0.96 \mathrm{~g} / \mathrm{cm}^{3}\right)$, with a 3.2-mm-diameter hole drilled parallel to the central axis at a radial offset of $10 \mathrm{~mm}$. A flexible tube filled with ${ }^{18} \mathrm{~F}$ solution was inserted into the 3.2-mm hole of the phantom. The initial activity was measured to be $18.8 \mathrm{MBq}$ using a dose calibrator (Atomlab 300; Biodex Medical Systems) at the start of the acquisition. The phantom was centered in the FOV and rotated by $45^{\circ}$ to achieve a more symmetric source distribution that also more closely represents the expected spatial distribution in mouse studies. A life-supporting chamber was included in the FOV for this measurement, creating a more realistic scatter environment.

The data were processed as specified by NEMA NU 4. The scatter fraction was measured using a prompt sinogram with an activity of $185 \mathrm{kBq}$. This low-activity frame was chosen to ensure that dead time and randoms did not affect the measurement. The scattered count rate was then calculated by Equation 1:

$$
R_{\text {scatter }}=R_{\text {prompt }}-R_{\text {true }}-R_{\text {random }},
$$

where $R_{\text {scatter }}, R_{\text {prompt }}, R_{\text {true }}$, and $R_{\text {random }}$ are the scatter, prompt, true, and random event rates, respectively. The scatter fraction (SF) was calculated by Equation 2:

$$
\mathrm{SF}=\frac{R_{\text {scatter }}}{R_{\text {scatter }}+R_{\text {true }}} .
$$

The noise-equivalent count rate (NECR) for each prompt sinogram was determined using the following equation:

$$
\mathrm{NECR}=\frac{R_{\text {true }}^{2}}{R_{\text {prompt }}+R_{\text {random }}}=\frac{\left(R_{\text {prompt }}-R_{\text {random }}\right)^{2} \times(1-\mathrm{SF})^{2}}{R_{\text {prompt }}+R_{\text {random }}} .
$$

\section{CT Subsystem Performance}

For the CT subsystem, we measured noise, dose, contrast, and spatial resolution. First, a $25-\mathrm{mm}$-diameter plastic cylinder filled with water was imaged, and noise was calculated as the ratio of the SD over the average value in a 4,000- $\mathrm{mm}^{3}$ uniform region. Dose was measured by placing a 10 -cm-long, pencil-shaped ionization chamber, model 10X5-3CT (Radcal Corp.), in a life-supporting chamber (including the cover) and acquiring a scan with the standard protocol. The $10-\mathrm{cm}$ wire was entirely in the $\mathrm{x}$-ray cone beam $(10.6 \mathrm{~cm})$. The dose was measured 5 times and averaged. For image contrast, a 25-mm-diameter acrylic glass phantom containing six 2-mm-diameter rods containing 4 iodine concentrations $(750,250,50$, and $0 \mathrm{mg} / \mathrm{mL})$ was scanned. The average image value in each rod was calculated and plotted against the nominal rod iodine concentration. Linearity was assessed by the coefficient of determination $\left(R^{2}\right)$ of a linear regression (ordinary least squares). Finally, resolution was measured with a micro-CT wire 
phantom (QRM GmbH) containing a $25-\mu \mathrm{m}$-diameter tungsten wire. The Fourier transform of a profile across the image (line-spread function) was used to calculate the modulation transfer function, and we report the resolution as the value of the modulation transfer function at $10 \%$ of the maximum.

\section{Imaging Studies}

NEMA Phantom Study. Image-quality studies were performed using the NEMA NU 4 image-quality phantom. The phantom (Data Spectrum Corp.) consists of 3 regions: a main fillable uniform region allowing the uniformity to be measured; a lid that attaches to the main fillable region, containing 2 smaller cold regions (filled with water and air) used to quantify the spillover ratio; and a solid acrylic region with 5 cylindric holes for measuring the recovery coefficients as a function of rod diameter. Because the G8 was designed for use with a low injection dose, the image-quality phantom was filled with $1.85 \mathrm{MBq}$ of ${ }^{18} \mathrm{~F}$ solution measured with a dose calibrator (Atomlab 300; Biodex Medical Systems). This activity is half the activity level recommended by the NEMA NU 4 protocol. The phantom was placed on a life-supporting chamber to simulate in vivo imaging, and data were acquired for $20 \mathrm{~min}$ as prescribed in the NEMA NU 4 protocol. Detector efficiency normalization and random event corrections were applied, but no scatter correction was applied. A CT scan of the phantom and its supporting bed was acquired, and the reconstructed CT image defining the data acquisition geometry was forward-projected through the PET system response matrix to generate attenuation correction of the PET data.

A 22.5-mm-diameter and 10-mm-high cylindric volume of interest was drawn over the center of the uniform region of the image-quality phantom. The average concentration values and SD in this volume of interest were measured to estimate the noise performance as a measure of uniformity. The image slices covering the central 10-mm length of the rods were averaged to obtain a single image slice of lower noise. Circular regions of interest were drawn in this image around each rod, with diameters twice the physical diameters of the rods. The maximum values in each of these regions of interest were measured and divided by the mean value obtained in the uniformity test to obtain the recovery coefficient for each rod size. The transverse image pixel coordinates of the locations with the maximum region-of-interest values were recorded and used to create 10-mm-long line profiles along the rods in the axial direction. The SD of the pixel values measured along each of these line profiles was calculated. Although no scatter correction was applied to the acquired dataset, the spillover ratio of the water- and air-filled coldregion chamber was calculated as specified in the NEMA NU 4 standard to provide a rough estimation of the scatter effects. The diameter of the volume of interest was $4 \mathrm{~mm}$ and encompassed the central $7.5-\mathrm{mm}$ length in the axial direction. The ratio of the mean in each cold region to the mean of the hot uniform area was reported as spillover ratio.

Mouse Study. The UCLA Animal Research Committee approved the animal studies, which were performed according to the guidelines of the Department of Laboratory Animal Medicine at UCLA. C57BL6 mice were anesthetized with $2 \%$ isoflurane in oxygen and injected via the tail vein with $2.78 \mathrm{MBq}$ of ${ }^{18} \mathrm{~F}$-FDG or $2.70 \mathrm{MBq}$ of ${ }^{89} \mathrm{Zr}$ anti-CD8 antibody. The animals were kept warm on heating pads throughout the imaging procedures. After $60 \mathrm{~min}\left({ }^{18} \mathrm{~F}-\mathrm{FDG}\right)$ or $6 \mathrm{~d}\left({ }^{89} \mathrm{Zr}-\right.$ anti-CD8 antibody) of tracer biodistribution, the mice were anesthetized and placed in dedicated G8 imaging chambers for PET/CT imaging. PET scans were acquired for $10 \mathrm{~min}\left({ }^{18} \mathrm{~F}-\mathrm{FDG}\right)$ or $20 \mathrm{~min}$ $\left({ }^{89} \mathrm{Zr}\right.$-anti-CD8) with an energy window of $350-650 \mathrm{keV}$ reconstructed using MLEM, followed by CT acquisition. All PET images were corrected for CT-based photon attenuation, detector normalization, and radioisotope decay (scatter correction was not applied) and converted to units of percentage injected dose per gram. Images were analyzed using AMIDE, version 1.0.4 (23), and OsiriX (http://www. osirix-viewer.com).

\section{RESULTS}

\section{Energy Resolution and Flood Histograms}

Supplemental Figures $1 \mathrm{~A}$ and $1 \mathrm{~B}$ show the flood images acquired from 2 detector modules in 1 panel (supplemental materials are available at http://jnm.snmjournals.org). For the crystals in all 4 panels, the measured energy resolution ranged from $15.0 \%$ to $69.6 \%$ FWHM, with a mean of $19.3 \% \pm 3.7 \%$. Supplemental Figure 1C shows a vertical profile across 1 column of crystals shown in Supplemental Figure 1A with a peak-to-valley ratio of 4.5, and Supplemental Figure 1D shows a horizontal profile across 1 row of crystals shown in Supplemental Figure 1B with a peakto-valley ratio of 4.8 .

\section{Spatial Resolution}

Intrinsic Spatial Resolution. The intrinsic spatial resolution of an opposing detector pair is shown in Figure 2. For crystal pairs along the detector short axis, the measured FWHM ranged from 1.16 to $1.41 \mathrm{~mm}$, with an average value of $1.30 \pm 0.08 \mathrm{~mm}$. For crystal pairs along the detector long axis, the measured FWHM ranged from 1.27 to $1.64 \mathrm{~mm}$, with an average value of $1.38 \pm$ $0.10 \mathrm{~mm}$

Image Spatial Resolution. Figures 3A and 3B show the reconstructed image spatial resolution in the radial, tangential, and axial directions measured in the central and one fourth axial transverse
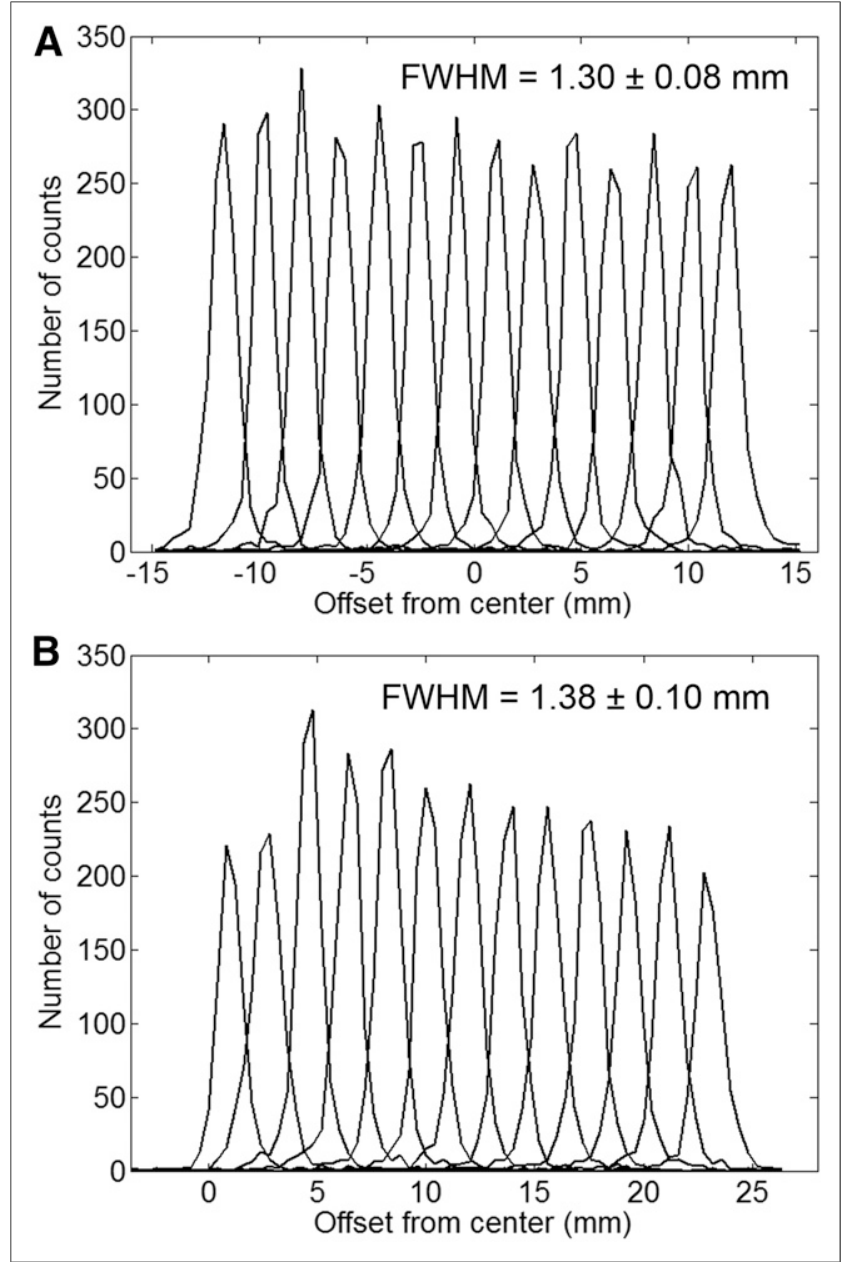

FIGURE 2. Intrinsic spatial resolution measured with ${ }^{22} \mathrm{Na}$ point source in direction along detector short axis $(A)$ and along detector long axis $(B)$. 


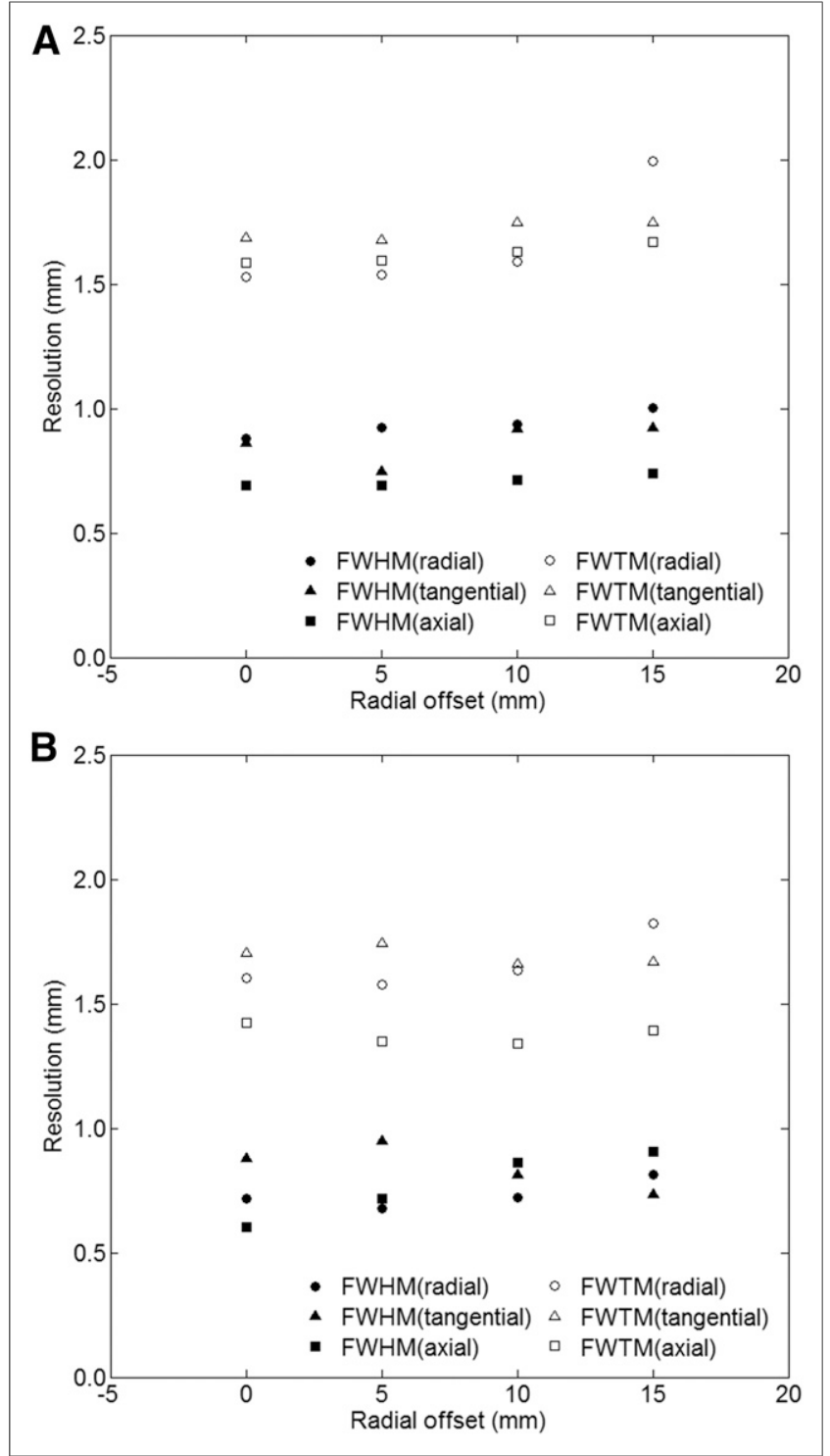

FIGURE 3. MLEM reconstructed image spatial resolution of G8 system, showing FWHM and full width at tenth of maximum (FWTM) of radial, tangential, and axial image resolution at axial CFOV (A) and $23.75 \mathrm{~mm}$ from axial CFOV toward axial edge of FOV (B).

plane, ranging from 0.60 to $1.01 \mathrm{~mm}$, with an average value of $0.81 \pm 0.11 \mathrm{~mm}$.

\section{Sensitivity}

Table 2 summarizes the absolute system sensitivity for different energy window settings at $10 \mathrm{~mm}$ from the axial CFOV toward the axial edge of the FOV, as well as for the axial CFOV. The maximum system sensitivity was $17.8 \%$ measured $10 \mathrm{~mm}$ from the axial CFOV and with a lower-level discriminator of $150 \mathrm{keV}$.

In previous work, a lower-level discriminator of $350 \mathrm{keV}$ was recommended as the optimized energy threshold for PET systems with detector geometries such as the G8 (17). With the optimal energy window of 350-650 keV, the G8 had a peak system sensitivity of $9.0 \%$ measured $10 \mathrm{~mm}$ from the axial CFOV. At the axial CFOV, the sensitivity was $8.6 \%$. The axial sensitivity profile with the energy window of $350-650 \mathrm{keV}$ is shown in Figure 4. The
TABLE 2

Absolute System Sensitivity as Function of Lower-Level Discriminator at $10-\mathrm{mm}$ Axial Offset and at Axial CFOV

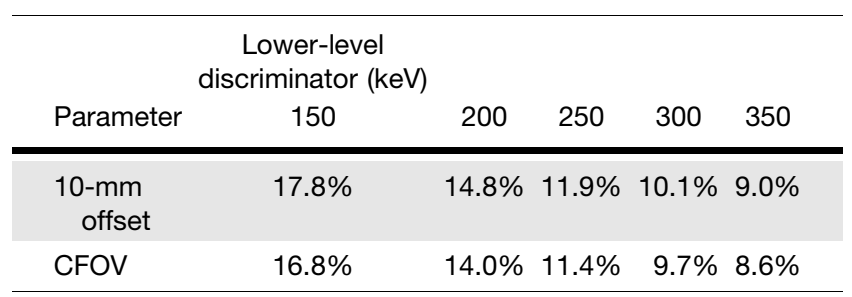

average sensitivity for a mouse-sized object (7-cm axial length) was $6.8 \%$.

\section{Scatter and Count-Rate Performance}

The prompt, random, and NECRs as a function of total activity in the mouse-sized phantom are plotted in Figure 5. The prompt rates peaked at around $3.6 \mathrm{MBq}$ of total activity. With this activity, the random events accounted for approximately $7 \%$ of the total prompt counts. The peak NECR was $44 \mathrm{kcps}$ achieved at a total phantom activity of $2.9 \mathrm{MBq}$. The scatter fraction for the mousesized phantom was $11 \%$.

\section{CT Subsystem Performance}

The calculated noise level in the uniform region of the water cylinder was $2.8 \%$. The average dose per scan was $50.1 \pm 1.2$ mGy. The G8 CT component does not have a mechanical shutter in front of the $\mathrm{x}$-ray source and therefore, while the system is waiting for the source voltage to ramp-up and the current to stabilize, some dose is given to the subject. This contribution was estimated to be about $7 \mathrm{mGy}$, or $14 \%$ of the total dose received. The contrast linearity (the CT value in the reconstructed image against the iodine concentration for each rod) was excellent both visually and as expressed by the coefficient of determination $\left(R^{2}=\right.$ 0.9998). The modulation transfer function (10\%) of the CT system was 2.05 cycles $/ \mathrm{mm}$. This value approximately corresponds to a FWHM of $0.4 \mathrm{~mm}$.

\section{Imaging Studies}

NEMA Phantom Study. Figure 6 shows the images (single slice, $0.46 \mathrm{~mm}$ thick) of a transverse plane of the uniform region, a coronal plane, a transverse plane with the 5 resolution recovery

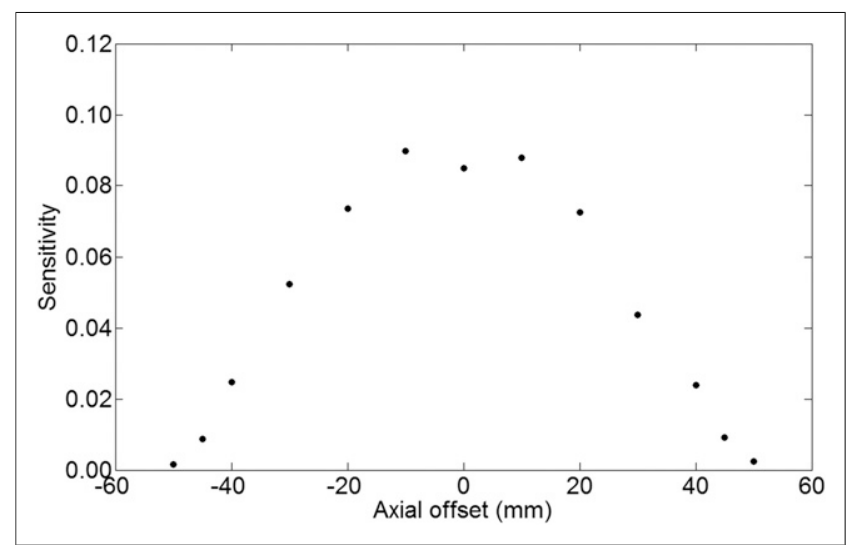

FIGURE 4. Absolute system sensitivity as function of axial position. Energy window was $350-650 \mathrm{keV}$. 


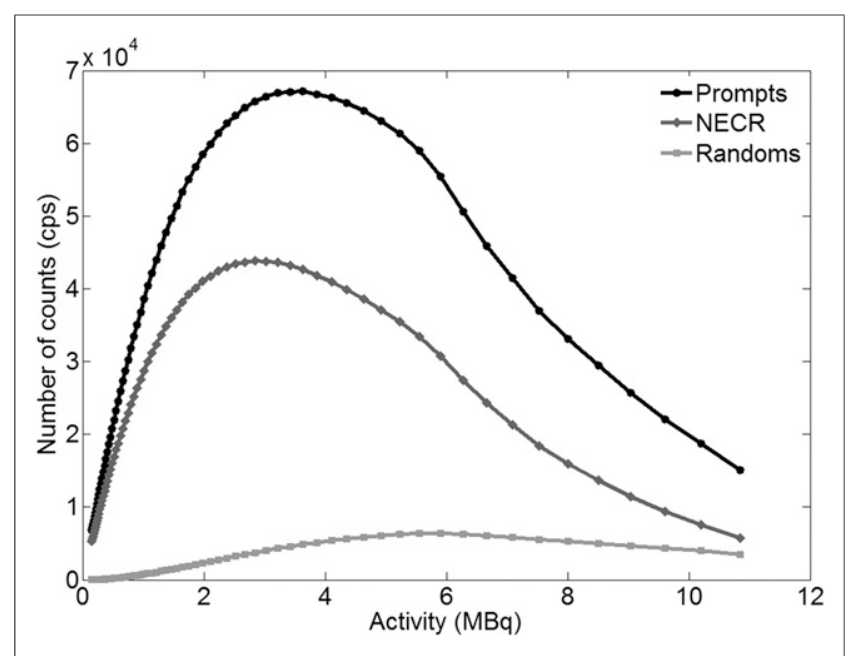

FIGURE 5. Count rate of G8 system as function of line source activity.

rods, and a profile across the uniform area of the NEMA imagequality phantom. With the MLEM reconstruction, the SD in the uniform region was $7.7 \%$. The recovery coefficients for the

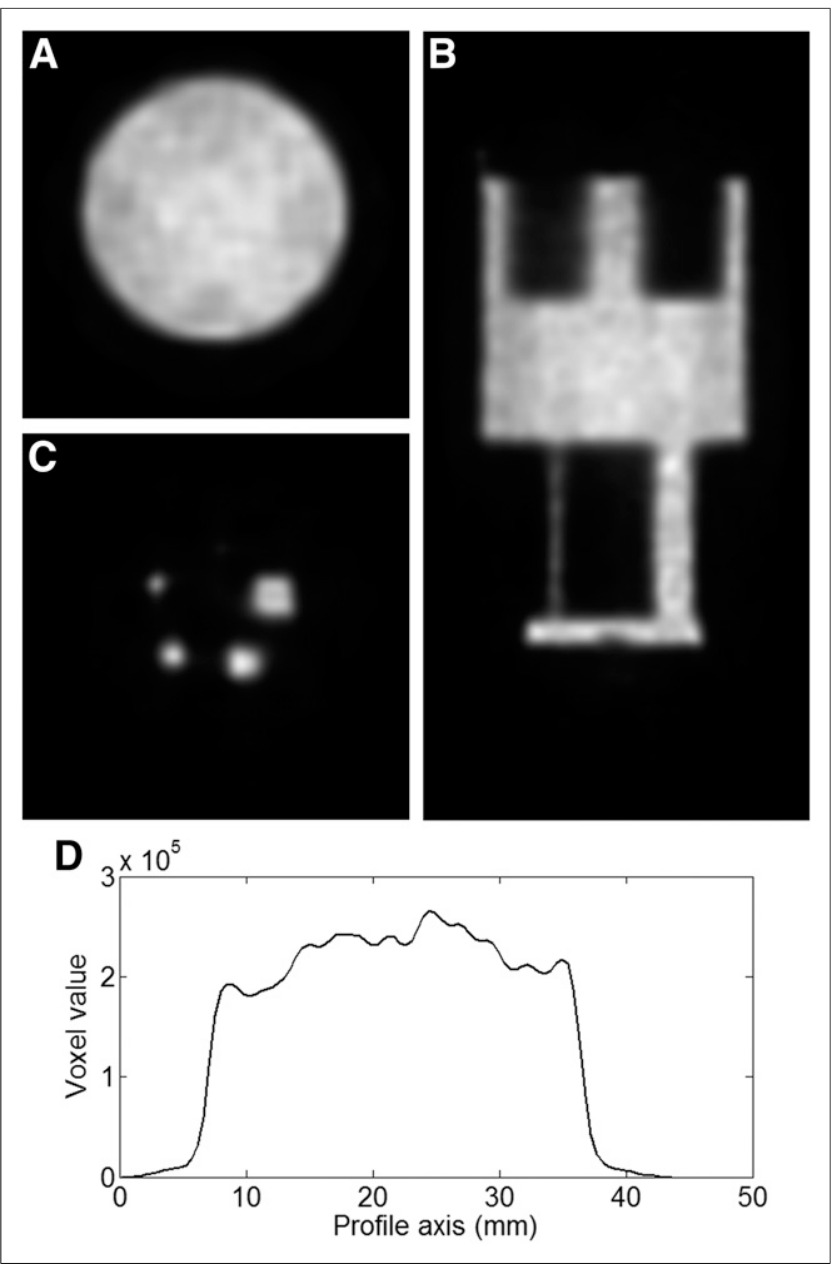

FIGURE 6. Reconstructed images of NEMA NU 4 image-quality phantom, filled with ${ }^{18} \mathrm{~F}(1.85 \mathrm{MBq})$ and imaged for $20 \mathrm{~min}$ : transverse slice of uniform region $(A)$; coronal slice $(B)$; transverse slice of rod region $(C)$; profile across uniform area (D).
5 different rod sizes from 1 to $5 \mathrm{~mm}$ in diameter were $0.11 \pm 0.01$, $0.70 \pm 0.08,1.07 \pm 0.09,1.10 \pm 0.11$, and $1.04 \pm 0.09$. The spillover ratios in the water- and air-filled cold-region chambers were $7.5 \% \pm 2.1 \%$ and $5.4 \% \pm 1.2 \%$, respectively.

Mouse Study. Representative images of the biodistribution of 2 PET tracers radiolabeled with different radioisotopes, ${ }^{18} \mathrm{~F}-\mathrm{FDG}$ and ${ }^{89} \mathrm{Zr}$-anti-CD8 antibody, are shown in Figure 7. Figure 7A shows coronal and sagittal images from a $10-$ min ${ }^{18} \mathrm{~F}-\mathrm{FDG}$ study on C57BL6 mice, distinguishing the myocardium from the blood pool and identifying other tissues such as the Harderian glands, brain including cerebellum, spinal column, intestines, kidneys, and bladder. The activity in the entire mouse was $1.96 \mathrm{MBq}$ at scan time. Figure 7B illustrates the capabilities of the PET/CT combination system in a study of immunocompetent C57BL6 mice injected with ${ }^{89} \mathrm{Zr}$-anti-CD8 antibody. A reconstructed 3dimensional volumetric PET image was registered with the CT dataset. The maximum-intensity-projection image of a 20-min scan shows well-defined mappings of the lymphoid organs spleen, thymus, and lymph nodes, particularly the axillary, brachial, cervical, inguinal, and popliteal lymph nodes. The expected biodistribution to the liver, kidneys, and bone was also observed. The activity in the entire mouse was $0.34 \mathrm{MBq}$ at scan time.

\section{DISCUSSION}

This investigation has evaluated the overall performance of the G8 PET/CT preclinical system. The PET component was evaluated according to the NEMA NU 4 standards with modifications as necessary. Noise, dose level, contrast, and resolution were evaluated for the CT component.

Besides an addition of 2 extra rows of crystals at the transverse edges of the FOV to reduce the gap between detectors, slightly different crystal dimensions, and segmented lightguides, a major

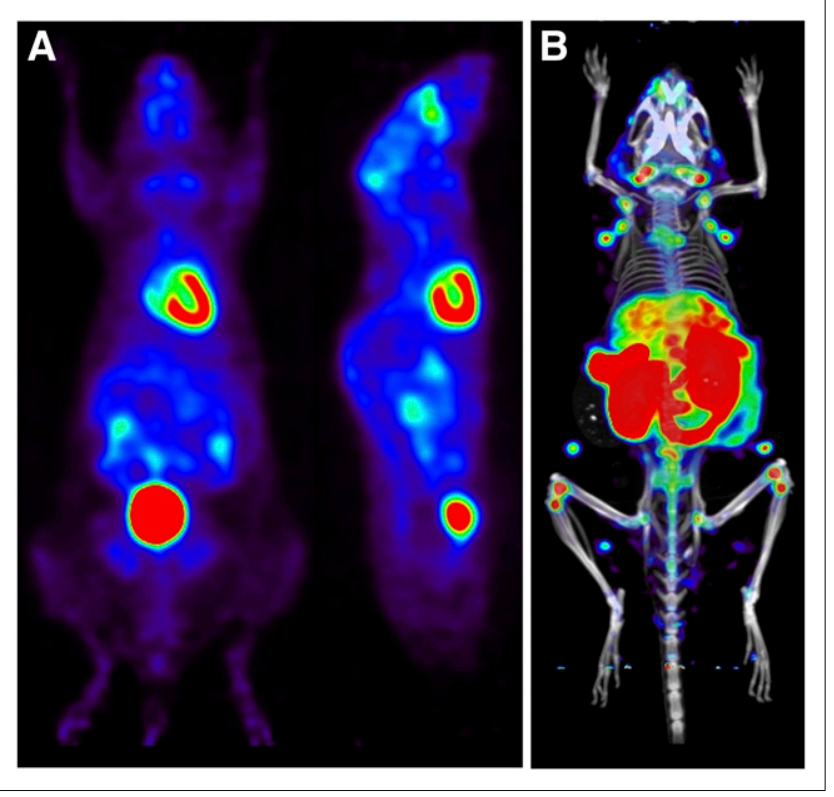

FIGURE 7. Reconstructed images of C57BL6 mice injected with ${ }^{18} \mathrm{~F}-$ FDG and ${ }^{89} \mathrm{Zr}$-anti-CD8 antibody. (A) 10-min static PET coronal and sagittal slices of mouse $60 \mathrm{~min}$ after injection of ${ }^{18} \mathrm{~F}-\mathrm{FDG}$ (1.96 MBq at scan time). (B) 20-min static PET/CT maximum-intensity projection of mouse $6 \mathrm{~d}$ after injection of ${ }^{89} \mathrm{Zr}$-anti-CD8 antibody ( $0.34 \mathrm{MBq}$ at scan time). 
change from the G4 to the G8 system is the data acquisition electronics used for detector signal digitization and processing. The G8 uses upgraded data acquisition electronics with twice the number of analog-to-digital converter channels. The outputs from each of the MAPMTs in 1 detector panel are separately multiplexed into 4 signals (energy, $x, y$, and dynode), and 8 analog-todigital converters are used to process the signals from each detector panel.

The increased granularity of the detector readout scheme in the G8 leads to an extended system dynamic range and better count rate performance. In the G4, the NECR peak was reached at lower total activity than for comparable instruments, because of its compact system geometry, the long decay time of BGO, and the multiplexed electronics $(12,15)$. For each $\gamma$-interaction in a detector module, the BGO signal is integrated for $800 \mathrm{~ns}$, during which no new event detection is possible. In the G8, when a $\gamma$-photon interacts with one of the 2 modules in a detector panel, the other module is still capable of event detection because the 2 modules in the panel are optically isolated and are read out separately. As a result, the activity at the NECR curve peak for the G8 is nearly double, at $2.9 \mathrm{MBq}$, the $1.5 \mathrm{MBq}$ for the $\mathrm{G} 4$. Because the design goal of the G8 has been high-sensitivity imaging of mice at a low injected dose, imaging with a dose higher than the NECR peak activity is not recommended. The detector would be saturated with pulse pileup, which may introduce image artifacts and compromise the spatial resolution. The system, though, can be used with much lower amounts of injected activity than are typical in the field (e.g., $0.34 \mathrm{MBq}$ of ${ }^{89} \mathrm{Zr}$, as shown in Fig. 7).

The new readout scheme also includes modifications of the detector and lightguide design. For each detector panel in the G4, 1 BGO scintillator array is coupled to 2 MAPMTs via $1 \mathrm{~mm}$ of clear glass. The scintillation light from crystals at the junction of the 2 MAPMTs is distributed to both MAPMTs. Light collection for these crystals is diminished because of the large insensitive area at the junction, degrading crystal identification and energy resolution. In the G8, each detector panel consists of 2 separate and optically isolated BGO scintillator arrays. Each array is coupled to the sensitive area of 1 MAPMT using a tapered, pixelated lightguide. As a result, the loss of light at the PMT junction area is reduced (24).

The sensitivity measured at the CFOV is lower than that at $\pm 10-\mathrm{mm}$ axial offsets, as shown in Figure 4, which also results from the new detector readout scheme used in the G8. When an annihilation $\gamma$-photon interacts via scattering in 2 crystal arrays within 1 detector panel, the energy deposited in each crystal array will be detected as a separate event. This increases the probability that neither of the 2 detections is qualified in the energy window of $350-650 \mathrm{keV}$. As a result, a fraction of the events happening at the edge crystal between the 2 detectors in 1 panel will be rejected, reducing the measured sensitivity at the CFOV. This loss of events could also be observed in the intrinsic spatial resolution result. The number of counts for the left 2 profiles in Figure 2B, which correspond to the edge crystals close to the junction of 2 crystal arrays, is lower than that for other profiles. The use of measured sensitivity at the CFOV $(8.6 \%)$ as the system peak sensitivity significantly underestimates the G8 system performance. The sensitivity for mouse imaging $(6.8 \%)$, averaged from the 7-cm axial length of the sensitivity profile, is a more suitable parameter and compares favorably with other systems (25). For the measured sensitivity reported in this work, the attenuation of the steel material surrounding the source was not compensated for.
The MLEM-reconstructed-image spatial resolution shown in Figure 3 is fairly homogeneous within the entire FOV, because the flat-panel boxlike geometry of the G8 leads to depth of interaction errors that are more uniformly distributed in the FOV. Figure 3 also demonstrates that the implemented MLEM reconstruction accurately models the physical response of the scanner in the system matrix, despite the fact that the detection of coincidences along very oblique angles is allowed because of the close geometry of the scanner. The image spatial resolution averaged $0.8 \mathrm{~mm}$ FWHM, as is consistent with the choice of the reconstructed image voxel size $(0.46 \times 0.46 \times 0.46 \mathrm{~mm})$, showing that the reconstruction is implemented properly. However, this value is not representative of the actual image spatial resolution of the system during in vivo imaging, since the method of measuring the image spatial resolution defined in the NEMA NU 4-2008 standard is incompatible with the iterative reconstruction algorithm. Because the G8 system does not provide traditional filtered backprojection image reconstruction, we report here the iterative image reconstruction results as a reference. For in vivo studies, the crystal size and the detector intrinsic spatial resolution $(\sim 1.4 \mathrm{~mm})$ are better indicators of the reconstructed image spatial resolution.

\section{CONCLUSION}

This study evaluated the performance of the G8 integrated PET/ CT system. With an energy window of 350-650 keV, the peak absolute sensitivity was $9.0 \%$ near the CFOV, and the average sensitivity for imaging a mouse-sized object (7-cm axial length) was $6.8 \%$. The total activity at the peak NECR for the mousesized phantom was $2.9 \mathrm{MBq}(78 \mu \mathrm{Ci})$. The dynamic range of the G8 is significantly better than that of the previous-generation G4. The overall performance demonstrates that the G8 scanner is suitable for producing high-quality images for molecular imagingbased biomedical research.

\section{DISCLOSURE}

Nam T. Vu and Zheng Gu are currently employees of Sofie Biosciences, Inc. Nam T. Vu and Arion F. Chatziioannou are shareholders of Sofie Biosciences, Inc. This work was supported by the UCLA Foundation from a donation made by Ralph and Marjorie Crump for the UCLA Crump Institute for Molecular Imaging and in part by the National Cancer Institute P30 CA016042 Cancer Center Support Grant. No other potential conflict of interest relevant to this article was reported.

\section{ACKNOWLEDGMENTS}

We thank Richard Tavaré and Kirstin Zettlitz from the Anna Wu Lab at the Crump Institute for Molecular Imaging at UCLA for providing the ${ }^{89} \mathrm{Zr}$-anti-CD8 antibody, and we thank the staff of the UCLA Ahmanson Biomedical Cyclotron Facility for providing ${ }^{18}$ F-ion and ${ }^{18}$ F-FDG. We also thank Waldemar Ladno and Olga Sergeeva of the Crump Institute's Preclinical Imaging Technology Center for their assistance with the imaging for this study.

\section{REFERENCES}

1. Phelps ME. Positron emission tomography provides molecular imaging of biological processes. Proc Natl Acad Sci USA. 2000;97:9226-9233.

2. Gambhir SS. Molecular imaging of cancer with positron emission tomography. Nat Rev Cancer. 2002;2:683-693. 
3. Myers R. The biological application of small animal PET imaging. Nucl Med Biol. 2001;28:585-593.

4. Chatziioannou AF. Molecular imaging of small animals with dedicated PET tomographs. Eur J Nucl Med Mol Imaging. 2002;29:98-114.

5. Townsend DW. Dual-modality imaging: combining anatomy and function. $J$ Nucl Med. 2008;49:938-955.

6. von Schulthess GK, Schlemmer HPW. A look ahead: PET/MR versus PET/CT. Eur J Nucl Med Mol Imaging. 2009;36(suppl):S3-S9.

7. Clark DP, Badea CT. Micro-CT of rodents: state-of-the-art and future perspectives. Phys Med. 2014;30:619-634.

8. Kinahan PE, Townsend DW, Beyer T, Sashin D. Attenuation correction for a combined 3D PET/CT scanner. Med Phys. 1998;25:2046-2053.

9. Watson CC, Casey ME, Michel C, Bendriem B. Advances in scatter correction for 3D PET/CT. IEEE Nucl Sci Symp Conf Rec. 2004:3008-3012.

10. Erlandsson K, Buvat I, Pretorius PH, Thomas BA, Hutton BF. A review of partial volume correction techniques for emission tomography and their applications in neurology, cardiology and oncology. Phys Med Biol. 2012;57:R119R159.

11. Wang H, Stout DB, Chatziioannou AF. Estimation of mouse organ locations through registration of a statistical mouse atlas with micro-CT images. IEEE Trans Med Imaging. 2012;31:88-102.

12. Gu Z, Taschereau R, Vu NT, et al. NEMA NU-4 performance evaluation of PETbox4, a high sensitivity dedicated PET preclinical tomograph. Phys Med Biol. 2013;58:3791-3814.

13. Herrmann K, Dahlbom M, Nathanson D, et al. Evaluation of the Genisys4, a bench-top preclinical PET scanner. J Nucl Med. 2013;54:1162-1167.

14. NEMA Standards Publication NU 4-2008: Performance Measurements of Small Animal Positron Emission Tomographs. Rosslyn, VA: National Electrical Manufacturers Association; 2008:23.
15. Gu Z, Prout DL, Taschereau R, Bai B, Chatziioannou AF. A new pulse pileup rejection method based on position shift identification. IEEE Trans Nucl Sci. 2016;63:22-29.

16. Douraghy A, Rannou FR, Silverman RW, Chatziioannou AF. FPGA electronics for OPET: a dual-modality optical and positron emission tomograph. IEEE Trans Nucl Sci. 2008;55:2541-2545.

17. Gu Z, Bao Q, Taschereau R, Wang H, Bai B, Chatziioannou AF. Optimization of the energy window for PETbox4, a preclinical PET tomograph with a small inner diameter. IEEE Trans Nucl Sci. 2014;61:1164-1173.

18. Mumcuoglu EU, Leahy R, Cherry SR, Zhou ZY. Fast gradient-based methods for Bayesian reconstruction of transmission and emission PET images. IEEE Trans Med Imaging. 1994;13:687-701.

19. Taschereau R, Rannou FR, Chatziioannou AF. A modeled point spread function for a noise-free system matrix. IEEE Nucl Sci Symp Conf Rec. 2011:4102-4105.

20. Feldkamp LA, Davis LC, Kress JW. Practical cone-beam algorithm. J Opt Soc Am A Opt Image Sci Vis. 1984;1:612-619.

21. Suckow C, Kuntner C, Chow P, Silverman R, Chatziioannou A, Stout D. Multimodality rodent imaging chambers for use under barrier conditions with gas anesthesia. Mol Imaging Biol. 2009;11:100-106.

22. Chow PL, Stout DB, Komisopoulou E, Chatziioannou AF. A method of image registration for small animal, multi-modality imaging. Phys Med Biol. 2006;51: 379-390.

23. Loening AM, Gambhir SS. AMIDE: a free software tool for multimodality medical image analysis. Mol Imaging. 2003;2:131-137.

24. Gu Z, Prout DL, Valenciaga Y, Chatziioannou AF. Lightguides for improving edge crystal identification and energy resolution in pixelated scintillator detectors. IEEE Nucl Sci Symp Conf Rec. 2015:1-3.

25. Goertzen AL, Bao QN, Bergeron M, et al. NEMA NU 4-2008 comparison of preclinical PET imaging systems. J Nucl Med. 2012;53:1300-1309. 